\title{
Future Neurology: 1 year on, and forging further into the future
}

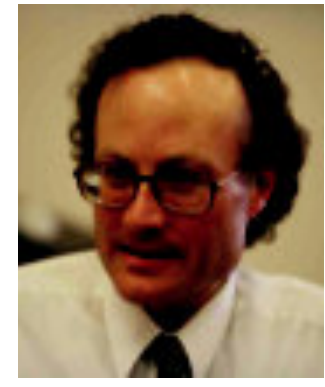

David Blacker

Sir Charles Gairdner Hospital, Department of Neurology \& Clinical Neurophysiology, Hospital Avenue, Nedlands, Western Australia \& University of Western Australia, Australia Tel.: +61 893463 088; Fax: +61 893462455 ; davidblackermd@ hotmail.com
'Future Neurology is ideally positioned to ride the wave of change and perhaps to assist with its advancement, disseminating new breakthroughs and stimulating its readers to reach new and greater heights in research and clinical practice.'

The January 2007 edition marks the 1 year anniversary of Future Neurology. This provides the opportunity to briefly review the highlights of the first year, but more importantly to prognosticate on the future. As a medical specialty, neurology has undergone a major transformation within the working lifetime of many of its current practitioners. From a somewhat arcane and mainly diagnostic discipline with few (if any) active treatments to offer, it is now a dynamic, rapidly changing field with a burgeoning array of diagnostic tools and new therapies for previously untreatable conditions. Thrombolytic therapy for stroke, interferons for multiple sclerosis (MS), cholinesterase inhibitors for Alzheimer's disease (AD) and deep brain stimulation for movement disorders are but a few obvious examples. Neuroimaging techniques and the concept of studying a disease at its genetic and molecular level mean we now have a much greater understanding of diseases than ever before. Many further advances are likely to come soon, benefiting from the global explosion of communication and information technology. Future Neurology is ideally positioned to ride the wave of change and perhaps to assist with its advancement, disseminating new breakthroughs and stimulating its readers to reach new and greater heights in research and clinical practice.

The emphasis of the journal is on the recent, the present and tomorrow, in particular highlighting new molecular approaches to advancing neurotherapeutics. New strategies to combat old foes, such as stroke, epilepsy, MS, Parkinson's disease $(\mathrm{PD})$ and $\mathrm{AD}$, are discussed in virtually every issue and, gratifyingly, new advances can be reported regularly. Its format matches the ethos of Future Neurology - punchy, pithy articles for the 'information overloaded' and time-poor clinician or researcher. The Bulletin Board, with Priority Paper Alerts, and Conference Scene articles, provide up-to-the-minute information that can be easily digested. The emphasis on therapeutics is highlighted by the Drug Evaluations. These have covered a broad range of neurological and psychiatric treatments for our bread-and-butter diseases such as migraine, epilepsy, neuropathic pain, depression and schizophrenia. In addition to specific therapies, one highlighted article has focused on neurotherapeutic strategy [1]. This suggests how the clinical pharmacological approach to prescribing antiepileptic drugs might be applied to the drug treatment of other neurological conditions; which may prove to be a very valuable way of thinking. Old ideas may prove to have new applications in the future.

Genetics has deservedly received great attention during the first year, including prospects for therapy in $\mathrm{AD}$ [2], brain tumors [3] and epilepsy [4]. Gropman's review was one of the highlights in the genetics coverage, emphasizing the concept of taking new discoveries from the bench-tobedside [5]. The application of genetic markers in $\mathrm{PD}$ and other articles on genetics are to be covered in the near future. Stem cell therapy for PD [6] and MS [7] has also been covered, and, no doubt, there will be much more on this to follow for these and other conditions.

The tremendous impact of neuroimaging has also featured highly, including; a glimpse into the next decade of structural imaging of patients with cognitive impairment [8], functional imaging in patients with MS [9], new insights into the imaging of pain pathways [10] and the role of nuclear imaging in the development of drug therapies for PD [11]. Further exciting applications of neuroimaging for stroke and prenatal brain injury are to be presented soon. Future Neurology reviews are characterized by conciseness and a slant on future perspectives. The comments within the bibliography of the reviews is a valuable and unique feature that encourages the reader to look further. A broad range of topics have been covered, from the molecular and experimental animal levels through to diagnostic approaches and therapeutics at population levels. Classic futuristic topics that have roots in current research have been covered, 
for example, bone marrow transplantation for a variety of neurological conditions [12] and olfactory ensheathing cells for the repair of spinal cord injury [13].

\section{'Future Neurology reviews are characterized by conciseness and a slant on future perspectives'.}

The perspectives section has provided not only a glimpse into future treatment of common conditions such as migraine [14] and $\mathrm{AD}$ [15], but has also given us a taste of how we might use the field of neuroinformatics at the bedside to translate the explosion of new neurological research data into a readily useable format to assist with patient care [16]. Indeed, the goal of neuroinformatics; 'to combine research in neuroscience with informatics to develop tools and approaches that will be essential for understanding the structure and function of the brain', is not dissimilar to the goal of Future Neurology. The September perspective entitled ' Use of complementary and alternative medicine for the treatment of children with cerebral palsy' [17] reminds us all that our approach to considering neurological disease must not only have an eye to the future, but it must also be an open-minded one.

In the future, the editors and editorial board will continue to strive to rapidly bring crucial neuroscience research to the reader. The international representation on the editorial board should help to ensure that a balanced, global view on developments in the field is maintained. This style of journal should be seen as an inspiration to the reader, rather than a bland report of new facts. In our second year, Future Neurology, will have further exciting articles on new diagnostic techniques, new treatments and, most importantly, new ways to think about our approach to neurological disease. The possibilities are limited only by our imaginations; although the reality may be limited by factors such as time and resources, there is surely no harm for a forum such as Future Neurology to foster a positive and encouraging attitude towards what lies ahead. The future has much to behold, with likely developments ranging from the genetic and molecular level for the individual patient, through to global strategies influencing the health of entire populations.

Never before has there been the opportunity to rapidly disseminate knowledge and to communicate advances with colleagues and the public so quickly after they occur. However, there is a responsibility for leaders in the field to ensure that dissemination of advances is accurate, balanced and tempered with the wisdom of experience; publications like Future Neurology must bear this in mind and show leadership in the exciting times that lie ahead. In recognizing the work of cuttingedge researchers and clinicians, we hope to stimulate the readers of Future Neurology to return invigorated to the bench or bedside, to fight for the future of our patients.

\section{Bibliography}

1. Eadie M: Remediable shortcomings in applying clinical pharmacology to neurological practice. Future Neurol. 1(6), 747-753 (2006).

2. Blesch A: Neurotrophin gene therapy for Alzheimers disease. Future Neurol. 1(2), 179-187 (2006).

3. Lesniak M: Neurosurgical oncology: current and future prospects. Future Neurol. 1(2), 127-129 (2006).

4. Kokaia M, Cecilia L: Neuropeptide gene therapy for epilepsy: viral vectors, stem cells and neurogenesis. Future Neurol. 1(6), 843-851 (2006).

5. Gropman A: Applying advances in neurogenetics to medical practice. Future Neurol. 1(5), 677-685 (2006).

6. Ben-Hur T: Human embryonic stem cell therapy for Parkinson's disease. Future Neurol. 1(2), 227-236 (2006).

7. Libbey JE, Tsunoda I, Fujinami RS: Autologous hematopoietic stem cell transplantation: a cure for multiple sclerosis? Future Neurol. 1(4), 403-408 (2006).
8. Testa C, Caroli A, Roberti V, Frisoni GB: Structural brain imaging in patients with cognitive impairment in the year 2015. Future Neurol. 1(1), 77-86 (2006).

9. Massimo F, Rocca M: Functional magnetic resonance imaging and multiple sclerosis. Future Neurol. 1(1), 67-76 (2006).

10. Davis KD: Recent advances and future prospects in neuroimaging of acute and chronic pain. Future Neurol. 1(2), 203-213 (2006).

11. Brooks DJ: Role of imaging in drug development for Parkinsons disease. Future Neurol. 1(3), 335-342 (2006).

12. Kentaro M: Future prospects of transplantation therapy for neurological diseases using adult bone marrow stromal cells. Future Neurol. 1(2), 215-226 (2006).

13. Harvey AR, Plant GW: Olfactory ensheathing glia and spinal cord injury: basic mechanisms to transplantation. Future Neurol. 1(4), 453-463 (2006).
14. Goadsby PJ: New targets in acute migraine treatment. Future Neurol. 1(2), 171-177 (2006).

15. Schulman H: Biomarkers to advance drug development for Alzheimers disease. Future Neurol. 1(4), 409-415 (2006).

16. Villoslada P, Oksenberg JR: Neuroinformatics in clinical practice: are computers going to help neurological patients and their physicians? Future Neurol. 1(2), 159-170 (2006).

17. Russman BS: Use of complementary and alternative medicine for the treatment of children with cerebral palsy. Future Neurol. 1(5), 545-551 (2006).

\section{Affiliation}

- David Blacker, FRACP Sir Charles Gairdner Hospital, Department of Neurology \& Clinical Neurophysiology, Hospital Avenue, Nedlands, Western Australia \& University of Western Australia, Australia Tel.: +61893463 088; Fax: +61 893462455 ; davidblackermd@hotmail.com 